\title{
Efficacy of Botulinum Toxin Stereotype A and Its Relevant Mechanisms of Action in Both Cosmetic and Therapeutic Uses
}

\author{
Orien L Tulp, ${ }^{1,2 *}$ Charles Poku Mensah, ${ }^{1}$ Rowany T Kakwere ${ }^{1}$ \\ ${ }^{1}$ University of Science Arts and Technology, Montserrat, BWI, USA \\ ${ }^{2}$ University of Health and Humanities, BVI, USA
}

\section{Section I}

Overview of Botulinum Toxin, Onabotulinumtoxina $\left(\right.$ Botox $\left.^{\oplus}\right)$ Actions and Applications

Keywords: Botox, Acetylcholine, Neuromuscular blockade

\section{Introduction and Overview}

Onabotulinumtoxin A (Botox®) is a parasympathetic autonomic blocking agent which acts as an inhibitor of the neuromuscular transmitter acetylcholine release and as a neuromuscular blocking agent, thereby inducing relaxation of selected muscle fibers for a duration of up to approximately 3 months duration. First approved by the FDA in 1989 and approved for further applications since 1991, the drug has now gained wide acceptance in cosmetic and other therapeutic applications, where the autonomic neurotransmitter acetylcholine blockade can bring about favorable cosmetic responses, particularly in the field of dermatology and cosmetic medicine. Since the original application of Onabotulinumtoxin A was approved for clinical use as a cosmetic agent, numerous additional applications have been approved by the FDA, all of which involve acetylcholine-mediated neuromuscular actions. The Onabotulinumtoxin A drug was derived from botulinum toxin B of the highly toxic soil microorganism Clostridium botulinum, often found in newly tilled grounds and a great concern for the agricultural industry, where inadvertent botulinum toxin contamination of food- stuffs may occur. Since botulinum toxin is one of the most potent neurotoxins known, extreme precaution and care must be observed during its precise administration to ensure the material does not diffuse into the general circulation, where minute quantities could prove deleterious or potentially death should blood concentrations reach paralytic levels. Thus, the drug must be administered via precise localized microinjection directly in the vicinity of the affected neuromuscular junction of diagnostic interest.

Botox administration involves the precise injection of a small, minute amount of a naturally occurring bacterial toxin into a small area of a patient's body, where it typically brings about its desired cosmetic actions by blocking the ability of the injected muscles to contract, thereby producing a semi-permanent effect which typically lasts until the affected nerve endings can regrow new attachments onto the muscle. Neuromuscular recovery times averages approximately three months in most individuals, during which time the decreased or absent muscle contractions allow an attenuation or softening of wrinkle lines, with the result to help to make the affected location, usually facial, appear more youthful. The dosage and administration site can become critical however, as over usage or overdosage may produce some decreased ability to perform routine facial expressions such as smiling. In addition, should some of the agent diffuse beyond the intended neuromuscular site, it may introduce unwanted anatomic features depending

\begin{tabular}{|l|l|}
\hline \hline Quick Response Code: & *Corresponding author: Orien L Tulp, USAT, US Office, Aspen Business Park, 5400 Ward \\
Road, Suite 150, Arvada C0 80002, USA \\
Received: 18 December, 2021 Published: 27 December, 2021
\end{tabular}


on how the microinjections occur, where they are placed, and how much quantity of the onabotulinum agent is injected. Presently, there are three additional forms of the botulinum toxin available, including abo botulinum toxin A (Dysport), rima botulinum toxin $\mathrm{B}$ (Myobloc) and Inco botulinum toxin A (Xeomin). Because each of the second-generation agents is a slightly different drug in terms of dosage units, preferred applications, and other properties, they are not interchangeable. Current FDA approved applications include the following conditions:

a) Cervical dystonia, where neck muscles contract involuntarily causing pain and uncomfortable positions.

b) Lazy eye., where there is an imbalance in the ocular muscles responsible for positioning the eye.

c) Neurological muscle contractures, including cerebral palsy, which can cause a patient's limbs to pull in toward their center.

d) Hyperhidrosis, where excessive sweating occurs in the absence of environmental stimulation or physical exertion, previously treated by sympathectomy.

e) Chronic migraine, where a patient may experience migraine attacks more than 15 days a month

f) Bladder dysfunction, where urinary incontinence may be caused by an overactive bladder.

g) Eye twitching, blepharospasm, and strabismus, where unwanted twitching or contracture of muscles around the eye occurs.

Thus, the purpose of the current paper is to provide a general overview of botulinum toxin actions and applications and is divided into three sections: Section I above is an introduction and broad overview; Section II is devoted to general and cosmetic pharmacological actions, and section III to Botox applications for bladder dysfunction.

\section{Section II}

\section{Review of Clostridium Botulinum Toxin, Serotype A}

\section{Abstract}

Microbial toxins have been discovered as the primary virulence factor(s) for a variety of pathogenic bacteria. Clostridium Botulinum is an obligate anaerobic bacterial spore producing gram-positive bacilli which produces Botulinum toxin, a very well-known potent neurotoxin. The potent neurotoxin substance produced by Clostridium botulinum organisms is presumed released by the organisms when targeting other bacterial or host cells for defensive or attack purposes, but also has been found to possess one or more medical applications. Many of the Clostridium species are known to produce up to 7 toxin serotypes each of which are immunologically distinct: types A-G: (A, B, C1, D, E, F, and G), for the purpose of this review we will concentrate our discussion on serotypes $A, B, E>F$, $G$ which are mostly associated with human diseases and medical applications. The neurotoxins produced by the Clostridium bacilli is a single inactive polypeptide chain that is cleaved with the aid of tissue proteinases into an active di-chain molecule: a heavy chain $(\mathrm{H})$ and a light chain (L) held collectively by way of a single disulfide bond ("Botulism; Botulinum toxin," n.d.). Among the serotypes each the ability to demonstrate its own varied mechanisms of action and duration of effect, possessing the ability to alter the normal metabolism function of host cells with predictable deleterious effects. The medical engineering and the scientific community have now turned a virulence factor of pathogenic bacteria Clostridium Botulinum into a treatment. Through its mechanism of action on acetylcholine secretion and action the potent toxic neurotoxins of Clostridium Botulinum (botulism) can be used for both cosmetic and therapeutic applications. This review describes both cosmetic and therapeutically relevant mechanisms of action of Botulinum toxin.

Keywords: Clostridium botulinum neurotoxin, Microbial toxins, Polypeptide chain, Immunologically, Beauty cosmetic

Abbreviations: BTX-A: Botox A; Ach: Acetylcholine; PNS: Peripheral Nervous System; CNS: Central Nervous System

\section{Objective}

To summarize the mechanism of action of Botulinum toxin A and its uses for therapeutic and cosmetic purposes.

\section{Areas Covered}

This review covers the brief microbiology, pharmacology, and physiological mechanism of action of Clostridium botulinum toxin, efficacy of stereotype A that is medically available and its associated challenges in therapeutic applications. Newer approaches in the fight against aging, as an option for the aesthetics and beauty industry, and for the use of botulinum injectables in the cosmetic surgery treatment of aging.

\section{Introduction}

\section{Microbiology and pharmacology}

Botulinum toxin type A, one of the most potent natural neurotoxins known to humankind is produced by Clostridium botulinum bacterium. ${ }^{1}$ Clostridium botulinum is a gram-positive, spore-forming, obligate, anaerobic bacillus that is commonly found in soil throughout the world. ${ }^{1,2}$ There are eight antigenically distinct, but structurally and functionally similar cytoplasmic exotoxins produced by Clostridium botulinum, each having a molecular weight of about 150-kDa, of which seven exotoxins are known to be neurotoxic. $^{3}$ They are identified as serotypes A, B, C1, C2, D, E, F, and G. Serotypes A, B, E>F, G, are the ones most commonly associated with 
human illnesses, and, of these, neurotoxin serotype A is the most potent. Diseases of other mammals and birds are caused by the exotoxins C1, C2, and D., The spores, especially of serotypes A and $\mathrm{B}$, are heat stable and resist boiling for hours, particularly at high altitudes. The neurotoxins, however, are heat labile, and when they appear in food, cooking can denature them and render them inactive. Conversely, the neurotoxins are acid stable and thus can survive the acidic digestive medium of gastric enzymes. Bacterial germination and elaboration of exotoxins are inhibited at a pH of less than 4.5 , and alkali also readily destroys the exotoxins rendering them inactive. Foods containing toxins A or B may appear and taste as if it were normal or possibly only slightly spoiled. ${ }^{5}$ When tainted food is digested and the neurotoxins are absorbed, the neurotoxins disseminate hematogenously to peripheral cholinergic motor nerve endings, where they bind irreversibly to the presynaptic terminals and block the release of the neurotransmitter acetylcholine at the neuromuscular junction.

\section{Acetylcholine}

Acetylcholine one of the major neurotransmitter chemicals found in the body between the nerve synapses, or gaps, between nerve cells. Acetylcholine plays a key role in both the functions of the peripheral nervous system (PNS) and the central nervous system (CNS). ${ }^{6}$ Acetylcholine is a neuromuscular activator in the peripheral nervous system, and it is neurotransmitter in the autonomic nervous system. It is the most abundant neurotransmitter in the body and its activation in the body elicits contraction of skeletal muscles and also activates glandular functions in the endocrine system. Acetylcholine elicits quick actions and its actions do not persist for prolonged durations. As a result, acetylcholine actions are rapidly terminated by degradation postsynaptically by another chemical substance called cholinesterase. Acetylcholine being a neurotransmitter is the chemical when is released by a neuron, or nerve cell, which will send propagation signals to another neuron across the synapse. The neurotransmitter binds to receptors to affect how the signal is received. The main objective of the neurotransmitter is to either amplify or inhibit the signals sent between the neurons. ${ }^{6,7}$

\section{Molecular mechanism of action of botulinum toxins}

Botulinum Toxins are toxic substances that are produced by the Clostridium bacteria for release in targeting other bacterial or host cells. ${ }^{8}$ The neuromuscular mechanism of action of botulinum toxin produced by Clostridium botulinum bacteria blocks the actions of acetylcholine in the body in a dose related manner. Acetylcholine is the major neurochemical found between the nerve synapses, or gaps, between nerve cells and muscles and is a muscle activator in the peripheral nervous system and a neurotransmitter in the autonomic nervous system. ${ }^{6,7}$ When acetylcholine actions are blocked or inhibited at a neuromuscular junction, it may cause a temporal paralysis of the affected muscles in a dose dependent manner. ${ }^{8}$ When a small amount of botulinum toxin injected into a neuromuscular junction, it will bind to the presynaptic membrane, and block the neurotransmitter release, thereby failing to propagate a post synaptic nerve signal, the botulinum toxin binds exclusively to inhibit acetylcholine release at the peripheral cholinergic motor neuron endplate, paralyzing the involved muscles, therefore effecting its therapeutic and cosmetic uses in a carefully monitored and dose-dependent manner. ${ }^{8,9}$

\section{Mechanism of action of Botox A (BTX-A)}

The first step in Botox A (BTX-A) paralysis is its attachment to the cholinergic receptor sites on the cell membrane of a presynaptic motor nerve end plate. ${ }^{8,9}$ The binding domain of the type A toxin molecule appears to be the $100-\mathrm{kDa}$ heavy chain, which is selective for cholinergic motor nerve terminals. This is known as the binding step and takes approximately 30 minutes from the time of injection. The internalization or second step is an energy-dependent receptor-mediated endocytic process, in which the plasma membrane of the nerve cell is turned around the toxin-receptor complex, forming a toxin-containing vesicle inside the nerve terminal. ${ }^{9}$ This process is independent of the concentration of calcium and is partially dependent on nerve stimulation. After internalization, the disulfide bond is cleaved by an unknown mechanism, and the $50-\mathrm{kDa}$ light chain of the toxin molecule is released across the endosomal membrane of the endocytic vesicles into the cytoplasm of the nerve terminal. This is the third or translocation step. The fourth and final step is called the blocking step. It is the $50-\mathrm{kDa}$ light chain of a toxin molecule that appears to be the domain responsible for blocking the transmission of a nerve impulse. The 50-kDa light chain inhibits acetylcholine release by acting as a zinc-dependent endopeptidase deactivating specific components of the neuroexocytic mechanism. This is accomplished by enzymatically cleaving the $25-\mathrm{kDa}$ synaptosome-associated protein (SNAP-25), a cytoplasm protein required for the docking of acetylcholine vesicles on the inner side of the nerve terminal plasma membrane ${ }^{10}$. The specific proteolysis of SNAP-25 by the $50-\mathrm{kDa}$ light chain of BTX-A prevents the fusion of acetylcholine vesicles onto the nerve membrane, thereby impeding the exocytosis of acetylcholine and its release into the synaptic cleft, causing the affected nerve terminals to be incapable of stimulating muscle contraction. BTX-A, however, does not affect the synthesis or storage of acetylcholine, nor the conduction of electrical impulses. ${ }^{10}$

\section{Therapeutic Uses of Botulinum toxin}

Botox ${ }^{\circledR}$ is a trade name for botulinum toxin $A$, a pharmaceutical agent made from a neurotoxin production from the clostridium botulinum toxin. ${ }^{11}$ Botox ${ }^{\circledR}$ was first used in 1977 to treat strabismus and in 1989 FDA first approved the drug for this purpose ${ }^{11,12}$ It 
has since been used medically to treat certain muscular treatments and wrinkles removal in cosmetic applications by temporarily paralyzing the involved muscles. ${ }^{12}$

Botox $₫$ is also used therapeutically to treat conditions ranging from focal dystonia (Spasticity, Velocity-dependent increase in muscle tone) and non-dystonic disorders and disorders of localized muscle spasms and smooth muscle hyperactive disorders. ${ }^{10-12}$

\section{Cosmetic Uses of Botulinum toxin}

Botox ${ }^{\circledR}$ cosmetic (botulinum toxin serotype A) blocks neuromuscular transmission via binding to a receptor site on motor nerve terminals, getting into the nerve terminals, and inhibiting the discharge of acetylcholine. This inhibition occurs because the neurotoxin cleaves SNAP-25, a protein imperative to the positive docking and release of acetylcholine from vesicles based within nerve endings when injected intramuscularly at therapeutic doses. ${ }^{10-12}$ The typical duration of cosmetic use lasts for approximately 3 to 4 months, until the neuromuscular junction regenerates and recovers from the neurotoxin injury.

Cosmetic Botox ${ }^{\circledR}$ produces partial chemical denervation of the muscle leading to a localized discontinuation in muscle activity in a dose dependent manner. In April 2002 FDA approved Botox® Cosmetic use for the treatment of moderate to severe hyperkinetic facial lines (glabellar frown lines, crow's feet) in adult men and women, ages 65 and younger. ${ }^{12}$ The drug works by means of blocking nerve impulses to loosen up the contraction of the two main forehead muscle groups. These contractions are the cause of vertical wrinkles between the eyebrows. After Botox® cosmetic treatment, noticeable improved appearance including attenuation or disappearance of the wrinkles can be observed as early as day or two, and the cosmetic effects may last for several months up to four months duration.

\section{Conclusion Part I}

Through medical engineering by the scientific community virulence factor of pathogenic bacteria like Clostridium Botulinum has been turned into clinically useful therapeutic and cosmetic applications, via pharmacologic modulation of neurotransmitter activity at point specific neuromuscular junction locations, Inhibitory actions on acetylcholine secretion by this potent neurotoxin from Clostridium Botulinum is the cause for its cosmetic and therapeutic uses. One of the crucial anatomical signs of aging is acceleration of wrinkling of the facial features. It mostly occurs naturally over a period time and is recognizable by biochemical and physiologic changes that are enhanced through a variety of environmental and physiologic factors commonly associated with biological aging.

There are several other factors that can elicit characteristic folds, furrows, and creases of the face. These can include the con- stant pull and frequent constant positional pressure on the skin of the face especially during sleep, and continues facial movements precipitated by the contractions of the facial muscles of expression. In an attempt to indemnify these adversary signs of anatomical aging, stress, gravity, and negative emotions, comes Botulinum toxin injection type A. It has been used to suppress the unwanted negative and expressive forehead, "crow's feet" of the face by reversing the weakness of the hypertrophic muscles of facial expression and lend one the impression that it becomes possible 'turn back the time' at least in the eyes of the beholder.

\section{References}

1. Benedetto AV. The environment and skin aging. Clinics in Dermatology. 1998;16(1):129-139.

2. BOTOX® Injections in Plastic Surgery: Overview, Botulinum Toxin, Cosmetic BOTOXÂ® Injections of the Face. (n.d.).

3. BОТОХ® Injections in Plastic Surgery: Overview, Botulinum Toxin, Cosmetic BOTOXÂ® Injections of the Face. (n.d.).

4. Botulism; Botulinum toxin. (n.d.).

5. Botulism; Botulinum toxin. (n.d.).

6. Sara A Kaltreider, Robert H Kennedy, John J Woog, et al. Cosmetic and Clinical Applications of Botulinum Toxin-Correction. Archives of Ophthalmology. 2005;123(6):794.

7. Scott AB. Botulinum Toxin Injection into Extraocular Muscles as an Alternative to Strabismus Surgery. Ophthalmology. 1980;87(10):10441049.

8. Acetylcholine: Definition, Function \& Deficiency Symptoms - Video \& Lesson Transcript | Study.com. (n.d.).

9. Botulinum Toxin: Overview, History, and Mechanism of Action. (n.d.).

10. Wheeler A, Smith HS. Botulinum toxins: Mechanisms of action, antinociception and clinical applications. Toxicology. 2013;306:124-146.

\section{Section III}

\section{Use of Botulinum Toxin $\left(\right.$ Botox $\left.^{\odot}\right)$ in Primary Care Med- icine for Bladder Dysfunction}

\section{Abstract}

Clostridium botulinum is a motile, rod-shaped, gram-positive, anaerobic, spore-forming bacterium, and has the ability to produce the neurotoxin botulinum toxin. This neurotoxin can cause severe flaccid paralysis in humans and other animals due to muscle relaxation and is probably among the most potent and lethal toxins known to humankind, natural or synthetic. Better food canning techniques of agricultural products has led to a drop in the quantity of yearly cases of botulism to about 1,000 cases worldwide. On average, 110 botulism cases are reported in the USA each year. About $72 \%$ of these cases are attributed to infant botulism, close to $3 \%$ of these cases are wound botulism, and the remainder, about $25 \%$ of reported cases, are attributed to food-borne origin. The number of cases of infant botulism and food-borne botulism has transformed only a little in recent years. Nonetheless, the incidence of wound 
botulism has become increased, especially in California, where it has been associated with the abuse of black-tar heroin, which causes contaminated wounds at heroin injection sites. The therapeutic use of the neurotoxin botulinum began in the 1970s. Since then, the neurotoxin botulinum is being used in the treatment of many medical conditions, including for example, focal dystonic disorders, spasticity, blepharospasm, strabismus, underarm sweating, muscle pain disorders cosmetic frown lines and wrinkles, and others.

Keywords: Botox $\AA$, Botulinum toxin, Neurotoxin botulinum, Therapeutic uses of Botox $®$, BOTOX®, Bladder dysfunction, Overactive bladder

Abbreviations: BoNT-A/Botox $\AA / B O T O X ®$ : Neurotoxin Botulinum/Botulinum Toxin; USFDA/FDA: United States Food and Drug Administration; Ach: Acetylcholine; SNAP-25: Synaptosome Associated Protein; VAMP: Vesicle Associated Membrane Protein

\section{Objective}

To assess the therapeutic benefits of the botulinum toxin (Botox $($ ) in primary care medicine.

\section{Areas Covered}

This mini review covers the brief history of the neurotoxin botulinum, its associated adverse effects, its therapeutic benefits in primary care medicine and newer attempts in using Botox I in the treatment of other disorders including bladder dysfunction.

\section{Introduction}

\section{A brief history}

Clostridium botulinum is a motile, rod-shaped, gram-positive, anaerobic, spore-forming bacterium that has the ability to produce the neurotoxin botulinum. ${ }^{1}$ The recorded history of Clostridium botulinum began in the $1700 \mathrm{~s}$, before the identification of the microbial organism was discovered. Clostridium botulinum bacteria were first isolated in 1895. In 1944, Botulism was labeled as a food-poisoning disease that caused flaccid paralysis of the muscles in humans. Dr. Edward Schantz isolated a neurotoxin produced by the bacterium. From 1949 to the 1950s, it was discovered that the neurotoxin botulinum, named BoNT-A (Botox), blocks neuromuscular transmissions by blocking the release of acetylcholine from motor endplate nerve endings. Dr. Alan B. Scott used the botulinum toxin in 1980 to treat an eye deviation condition known as strabismus successfully. In December 1989, The U.S. Food and Drug Administration - (FDA), approved the use of Botox for the treatment of strabismus, blepharospasm, and hemifacial spasm in young patients. Botox for cosmetic purposes, i.e., to treat glabellar lines, also known as frown lines and wrinkles was approved in 2002 by the FDA. The FDA has since approved Botox for many additional uses since then. ${ }^{2}$

\section{Botulinum toxin Mechanism of action}

Botulinum toxin acts at high-affinity presynaptic acetylcholine binding recognition sites on the cholinergic nerve terminals, thereby lowering the quantity of acetylcholine released during neurotransmission, inflicting a dose-dependent neuromuscular blocking effect. This mechanism laid the basis for the progress of the toxin as a therapeutic tool. ${ }^{3}$ Healing occurs by way of proximal axonal sprouting of new synaptosome-associated vesicles and muscle re-innervation by means of formation of a newly regenerated neuromuscular junction over the course of approximately 4 months duration from the time of the neurotoxin intervention. ${ }^{4}$ BoNT-A and BoNT-E cleave a presynaptic membrane protein required for fusion of neurotransmitter-containing vesicles, i.e., synaptosome-associated protein (SNAP-25). ${ }^{5}$ BoNT-B, BoNT-D, and BoNT-F cleave synaptobrevin, a vesicle-associated membrane protein (VAMP). BoNT-C acts by cleaving a target membrane syntaxin protein. ${ }^{6,7,9}$

\section{Duration of botulinum toxin action}

Neurotoxin botulinum causes paresis in striate muscle within two to five days and lasts from two to three months before the pharmacologic effects it gradually begins to dissipate as the receptor end-plate regenerates over time. , $^{1,2,8}$

\section{What are the adverse effects of the botulinum toxin?}

Post-marketing reports point out that the effects of all botulinum toxin merchandise and Botox may spread from the site of injection to cause effects consistent with botulinum toxin effects. ${ }^{10}$ Adverse effects due to cosmetic and therapeutic injection of BoNT-A include ptosis, dysphagia, facial and other muscle weakness, respiratory problems, flu-like syndrome, seizure, and injection site and skin reactions. ${ }^{11}$ Of the 406 reports involving therapeutic use, 217 met the FDA's definition of serious, with 28 deaths and 17 seizures reported. ${ }^{12}$ Clinical findings submitted to the FDA for therapeutic cases differed from those of cosmetic BoNT-A cases, which were frequently less severe. Most of the unwanted effects were linked to local tissue diffusion of BoNT-A. Cautious attention to drug dose, accuracy in dilution preparations, care in handling and, storage, and site of injection including site preparation are required for optimal treatment end results and to lower the incidence antagonistic or adverse results, as human error is a common observation when adverse reactions are reported. ${ }^{9,12}$

\section{Clinical Applications-Bladder Dysfunction}

BOTOX® for injection can be used for the cure of severe bladder symptoms such as urinary incontinence as a result of detrusor overactivity related to neurological conditions, overactive bladder with signs of urge urinary incontinence, urgency, and frequency. ${ }^{13}$ It really works with the aid of blockading the ability of some nerves to better coordinate neural functions with sphincter or bladder mus- 
cles. It is used by medical specialties which include plastic surgery, urology, ophthalmology, colorectal surgery and orthopedics to treat specific medical conditions that are not responsive to other conservative therapies or medications. When treating patients with Botox® for urinary incontinence, the medical professional makes use of a small telescope called a cystoscope to visualize throughout the bladder interior and injects one 100 to 300 units of Botox® into the bladder wall. The procedure takes approximately 20 minutes and is usually carried out under general or local anesthesia, depending on the health care provider's choice. It is viewed as an outpatient procedure, which means that there is no expected hospitalization afterwards. Treatment with Botox® can be repeated when the benefits from the previous treatment have diminished, but there should normally be a minimum of 12 weeks between treatments to allow adequate recovery time. Botox's effectiveness and safety were established in two clinical trials of 1,105 individuals with symptoms of overactive bladder. Patients had been randomly assigned to obtain injections of a hundred units of Botox, twenty injections of five units each or placebo. Outcome after 12 weeks, it was confirmed that individuals treated with Botox experienced urinary incontinence an average of 1.6 to 1.9 fewer instances per day than individuals treated with placebo. Botox-treated individuals also needed to urinate on average 1.0 to 1.7 fewer times per day and expelled an average of about $30 \mathrm{cc}$ more urine per void than those treated with placebo. ("FDA approves Botox to treat overactive bladder," n.d.) ${ }^{14,15}$

The most commonly reported side effects reported for Botox treated overactive bladder within 12 weeks of injection included dysuria (BOTOX® 9\%, placebo 7\%), urinary tract infection (BOTOX® $18 \%$, placebo $6 \%$ ), bacteriuria (BOTOX® $4 \%$, placebo $2 \%$ ), residual urine volume (BОTOХ® three $\%$, placebo $0 \%$ ) and urinary retention $($ ВOTOX® $6 \%$, placebo $0 \%) .{ }^{16}$

\section{Discussion}

There had been spontaneous reports of loss of life, normally related to dysphagia, pneumonia, and/or different enormous debility or anaphylaxis, after cure with botulinum toxin. There have also been reports of adversarial pursuits involving the cardiovascular system, including arrhythmia and myocardial infarction, some with fatal results. A few of these patients had hazard risk factors together with pre-existing cardiovascular comorbidities. The certain relationship of these events to the botulinum toxin injection have not been conclusively established yet.

\section{Conclusion}

Botulinum toxin has revolutionized the treatment of distinctive cosmetic and therapeutic applications involving neuromuscular cholinergic transmission. For the primary care physician who treats individuals with complaints regarding Ach-mediated actions and refractory muscle spasticity, Botox presently provides the clinician an achievable and well-studied safe alternative to previously accepted standard of care solutions. Botox can be a potentially lethal neurotoxin, however, and should always be used with the utmost care and precaution. Botox use requires the provider to undergo specialized training and experience to safeguard against unanticipated adverse events, and care must be taken to prepare for the worst.

\section{Acknowledgments}

None.

\section{Funding}

None.

\section{Conflicts of Interest}

Author declares that there is no conflict of interest.

\section{References}

1. Botulinum Toxin in Primary Care Medicine. (n.d.).

2. Botulism Symptoms, Causes, Treatment - What causes botulism? Medicine Net. (n.d.).

3. Davis CP. Botulism: Symptoms, treatment \& medical uses for botulinum toxin.

4. Functional repair of motor endplates after botulinum neurotoxin types a poisoning: Biphasic switch of synaptic activity between nerve sprouts and their parent terminals. 1999.

5. Botulinum neurotoxin A selectively cleaves the synaptic protein SNAP-25.

6. Botulinum Toxin: Overview, History, Mechanism of Action. (n.d.).

7. Botulinum Toxin: Overview, History, Mechanism of Action. (n.d.).

8. Nickerson KW, De Pinto J, Bulla LA Jr. Lipid metabolism during bacterial growth, sporulation, and germination: kinetics of fatty acid and macromolecular synthesis during spore germination and outgrowth of Bacillus thuringiensis. Journal of bacteriology. 1975;121(1):227233.

9. Dressler D, Saberi FA, Barbosa ER. Botulinum toxin: mechanisms of action. Arq Neuro Psiquiatr. 2005;63(1):180-185.

10. About Cervical Dystonia | BOTOX® (onabotulinumtoxin A) for Medical Professionals. (n.d.).

11. Botulinum toxin type A injections: adverse events reported to the US Food and Drug Administration in therapeutic and cosmetic cases. (n.d.).

12. What are the reported adverse events due to therapeutic and cosmetic injection of botulinum toxin (BoNT)? (n.d.).

13. BOTOX Reference ID: (4263493). (n.d.).

14. FDA approves Botox to treat overactive bladder. (n.d.).

15. What are the FDA approved indications for botulinum toxin (BoNT)? (n.d.).

16. Allergan. BOTOX Onabotulinum toxin A Injection. 